RESEARCH

\title{
THE EFFECTS OF 1.5 TESLA CONTRAST- ENHANCED TEMPORAL BONE MRI ON THE AUDITORY FUNCTIONS AND COMPLAINTS OF GERIATRIC PATIENTS WITH TINNITUS
}

Turkish Journal of Geriatrics DOI: 10.31086/tjgeri.2020.158 2020; 23(2): 230-240

- Nesibe Gül YÜKSELASLIER ${ }^{1,2}$ (D)

- Özlem SAATÇ/2 (D)

- Sevim YILDIZ

CORRESPONDANCE

Nesibe Gül YÜKSELASLIER

Health Science University, Bursa Yuksek Ihtisas Training and Research Hospital, Department of Otorhinolaryngology, Bursa, TURKEY

Phone: +905554064764

e-mail: nesibe.gul.yuksel@gmail.com

Received: February 21, 2020

Accepted: May 14, 2020

${ }^{1}$ Health Science University, Bursa Yuksek Intisas Training and Research Hospital, Department of Otorhinolaryngology, Bursa, TURKEY.

${ }^{2}$ Sancaktepe Şehit Prof. Dr. Ilthan Varank Training and Research Hospital Department of Otorhinolaryngology, Istanbul, TURKEY.

${ }^{3}$ Sancaktepe Education and Research Hospital, Department of Radiology, Istanbul, TURKEY.

\section{Abstract}

Introduction: Temporary hearing threshold shift might develop after noise exposure due to magnetic resonance imaging. We aimed to investigate the effects of acoustic noise during 1.5 Tesla temporal bone MRI on audiometric tests and disturbance self-reports in patients with tinnitus.

Materials and Method: Sixty-three symptomatic ears of 55 patients with persistent tinnitus were included in this study. Sound level recordings of imaging room were made with dosimeter. Two age groups ( $<65$ years and $>65$ years) were created. Hearing thresholds were measured before, 24 hours after and 1 month after performing magnetic resonance imaging. Visual analogue scale, tinnitus handicap inventory and the Beck depression inventory were applied to all patients before and 24 hours after the imaging.

Results: The mean intensity of acoustic noise during imaging was recorded as $99.3 \pm 3.4 \mathrm{dBA}(109.9 \pm 4.1 \mathrm{~dB})$. The threshold shifts were statistically higher in patients aged $\geq 65$ than the ones aged $<65$ for 2000 and 4000 frequencies $(p<0.05)$. The mean temporary shifts in tinnitus loudness were $5.00 \pm 6.495 \mathrm{~dB}$ and $10.17 \pm 11.179$ $\mathrm{dB}$ for the patients with age $<65$ and age $\geq 65$ respectively $(p=0.018)$. While majority of the time dependent effects were significant for audiometric tests; they were insignificant for self-reported questionnaires, except visual analogue scale 5 , which was higher in patients aged $<65(p=0.012)$.

Conclusion: Acoustic noise due to 1.5 Tesla temporal bone magnetic resonance imaging caused hearing threshold shifts and deterioration in intensity and disturbance of the tinnitus especially in elderly. Hearing protection is essentially required for all patients, when it is indicated.

Keywords: Hearing Loss; Magnetic Resonance Imaging; Noise; Quality Of Life; Tinnitus. 


\section{INTRODUCTION}

Magnetic resonance imaging (MRI) is widely used in the differential diagnosis of auditory pathologies accompanied by tinnitus, especially in the presence of unilateral, asymmetrical, highfrequency sensorineural hearing loss. In spite of new technology, noise exposure in a high-intensity, wide-frequency range is still experienced in this imaging technique, which is used for the exclusive diagnosis of pathologies such as tumors located in the cerebellopontine angle and internal acoustic canal region (1).

High acoustic noise levels during MRI may temporarily disrupt hearing thresholds and could possibly cause a temporary threshold shift (TTS) that reverses after noise exposure is discontinued (2). The acoustic noise associated with MRI procedures is due to the rapid alterations of currents within the gradient coils. These currents produce significant acoustic noise that manifests as loud tapping, knocking, or chirping sounds and can cause temporary or, sometimes, permanent injury to the cochlea $(3,4)$. Several previous studies have reported an increase in MRI-induced hearing thresholds and even cases of hearing loss $(5,6)$. However, for patients using foam earplugs, the noise generated by a $3 \mathrm{~T}$ MRI procedure did not cause any TTS during high-frequency hearing measurements in another study (7).

During daily practice, we noticed that some geriatric patients reported that their tinnitus increased immediately after MRI. Therefore, we hypothesized that the auditory functions are disturbed and that complaints regarding tinnitus become more evident after 1.5 Tesla contrastenhanced MRI of the temporal bone.

The primary aim of this study was to determine the effects of acoustic noise during 1.5 Tesla contrast-enhanced temporal bone MRI on the intensity and frequency of tinnitus and degree of complaints, especially in geriatric patients.

\section{MATERIALS AND METHODS}

The present study was conducted in the Department of Otorhinolaryngology and Head \& Neck Surgery of a tertiary clinic after approval was received from the ethics committee of the local council (no. FSMEAH-KAEK 2019/1-18). The study was performed in accordance with the most recent version of the Helsinki Declaration. Written informed consent was obtained from all subjects included in the study.

\section{Patients}

All patients who presented with a complaint of tinnitus to the outpatient clinic of the otorhinolaryngology department of a tertiary center between February 2019 and August 2019 were prospectively included in this study. After the patients' medical history was taken, physical examinations regarding the ear, nose, throat, head, and neck were performed together with neurological examinations. Routine laboratory tests were conducted to rule out potentially treatable causes of tinnitus. Pure-tone audiometry (PTA) and tinnitus matching (TM) tests for pitch and loudness were used. Contrast-enhanced temporal bone MRI was performed in patients with tinnitus and asymmetric hearing loss of $10 \mathrm{~dB}$ or more compared with the other ear at frequencies of 4,000 to $8,000 \mathrm{~Hz}$.

\section{Inclusion criteria}

1. Individuals 18-75 years of age.

2. Patients with tinnitus who required investigation via MRI.

3. Patients who were fully capable of communicating with the researchers and obeying simple commands.

4. Patients admitted to outpatient clinics without any morbidity.

\section{Exclusion criteria}

1. Patients younger than 18 years and older than 76 years. 
2. Patients with MRI requested for reasons other than tinnitus.

3. Patients who were not capable of communicating with the researchers and obeying simple commands.

4. Patients with a history of inner ear pathology and ototoxic drug use.

5. Patients with a tumor in the cerebellopontine angle and internal acoustic canal, peripheral, or central vestibular disease, cardiovascular disease, previous head trauma, neurological disease, metabolic disease, or pregnancy.

\section{Bedridden patients.}

Initially, 101 patients, who had been free of medical agents that could cause tinnitus or had been prescribed for tinnitus were enrolled in the study. Two patients with vestibular schwannoma and six patients with vascular loop syndrome were excluded following examinations that revealed certain pathologies. One patient with a tinnitus frequency of $250 \mathrm{~Hz}$ and two patients with a tinnitus frequency of $500 \mathrm{~Hz}$ were also excluded to homogenize the high-frequency tinnitus sample of this research. Thereafter, 57 patients who met the above inclusion and exclusion criteria were selected for inclusion in the study.

\section{Contrast-Enhanced Temporal Bone MRI Procedure}

The MRI scan used in this study was performed by a device with a 1.5 Tesla magnetic field density (GE Healthcare SignaTM Explorer, United States). The standardized steps performed for MRI of the temporal bone were as follows: 1) localization (10 sn); 2) Ax T2 prop (full brain) (100 sn); 3) Cor 3D Fiesta (110 sn); 4) Ax 3D Fiesta (110 sn); 5) Ax T1 FSE (Thin) (90 sn); 6) Ax DWI b1000 Prop (185 sn); 7) Cor T1 FSE (90 sn); 8) +C Cor T1 FSE (90 sn); and 9) + C Ax T1 FSE (Thin) (118 sn). All MRI scans of the temporal bone lasted 15 minutes and 5 sn per session. The patients were scanned without any hearing protective equipment during MRI.

\section{Sound Pressure Level Measurements During the MRI Scan}

Average sound pressure level (SPL) measurements were gathered in both unweighted $(d B)$ and $A$-weighted $(d B A)$ decibels for the MRI scans. The sound level recordings were taken with a dosimeter containing a sound level meter and microphone (CEM DT 805 L, Shenzhen, China) that had a sound level measurement range of intensity 30-130 dB and frequency $31.5-8 \mathrm{kHz}$. The device was mounted within $50 \mathrm{~cm}$ of each patient's head position. The background noise in the room before and during the MRI scans was recorded by the calibrated dosimeter.

\section{Audiometric Tests}

The results of the PTA and TM tests for pitch and loudness were analyzed for the symptomatic ears. The PTA and TM test results were evaluated on the basis of test results on admission before the MRI (test 1), within 24 hours after the MRI (test 2 ), and 1 month after the MRI (test 3). Any change of $10 \mathrm{~dB}$ or more was accepted as a significant threshold shift.

\section{PTA}

The PTA tests were performed blindly in soundproof booths (AC40, Interacoustics, Middelfart, Denmark) and revealed air and bone conduction hearing thresholds at frequencies of 250, 500, $1,000,2,000,3,000,4,000$, and 8,000 Hz for each ear (on admission, within 24 hours after MRI, and 1 month after MRI). A threshold change above $10 \mathrm{~dB}$ from baseline recordings was considered significant to define TTS and permanent threshold shift (PTS).

\section{Tinnitus Pitch Matching and Tinnitus Loudness Matching}

The tinnitus severity and frequency pairing tests (on admission, within 24 hours after MRI, and 1 month after MRI) were performed blindly in sound-proof booths as well (AC40, Interacoustics, Middelfart, Denmark). Any frequency change and 
threshold change above $10 \mathrm{~dB}$ from the baseline record was considered significant.

\section{Self-Report Test for the Daily Life Effects of Tinnitus}

As tinnitus is a subjective phenomenon and difficult to evaluate; we analyzed disturbances caused by tinnitus according to the VAS, THI, and BDI scales upon admission before the MRI (test 1) and 24 hours after the MRI (test 2) for the symptomatic ears. The third test was not implemented, as 1 month would not have been suitable for relating the daily effects of tinnitus to MRI imaging via subjective self-reports.

\section{VAS Assessment for the Favourability of Tinnitus}

To understand the degree of tinnitus-related disturbance or daily life effects of tinnitus, the VAS was used upon admission before the MRI and 24 hours after the MRI (8). All patients were asked to rate their tinnitus from 0 (most favorable) to 10 (least favorable) for each of the five questions listed below:

VAS 1: Please mark the severity/intensity of your tinnitus.

VAS 2: Please mark the frequency/duration of your tinnitus.

VAS 3: Please mark the degree of disturbance caused by your tinnitus.

VAS 4: Please mark the degree of attention deficit caused by your tinnitus.

VAS 5: Please mark the degree of sleep problems caused by your tinnitus.

\section{THI for the Disability Induced by Tinnitus}

The validated Turkish version of the THI was used to address the degree of disability experienced by patients due to tinnitus upon admission before the MRI and 24 hours after the MRI (9). The THI was composed of 25 questions, each of which was scored on a scale of 0 to 4 . With respect to the functional, emotional, and catastrophic responses due to tinnitus, the minimum total score was 0 , and the maximum total score was 100. A test score of 0-16 meant "no or slight handicap", 18-36 meant a "mild handicap", 38-56 meant a "moderate handicap", 58-76 meant a "severe handicap", and 78-100 meant a "catastrophic handicap" (10).

\section{BDI for a Depressive Mood Related to Tinnitus}

The BDI was used to determine the extent of patients' depressive symptoms with tinnitus (11). This 21-question multiple-choice self-report inventory was given to the patients upon admission before the MRI and 24 hours after the MRI. The question scoring ranked the severity of each item from zero to three. A score of $0-13$ indicated minimal depression, 14-19 mild depression, 20-28 moderate depression, and 29-63 severe depression (11).

\section{Statistical Analysis}

The data in the present study were collected and analyzed by using SPSS 20.0 software package $($ IBM $®$ SPSS $®$ Statistics 20.0, Armonk, NY, U.S.A.). The Shapiro-Wilk test for skewness and kurtosis showed normally distributed data except for the PTA and TM threshold shifts. Numeric variables were presented as mean \pm standard deviation, and categorical variables were presented as frequency (n) and percentage (\%). The comparisons of the pre- and post-MRI PTA and TM thresholds and VAS, THI, and BDI scores within the same group were performed via a paired-sample t-test. Student's t test was used to compare the PTA and TM thresholds, and Mann-Whitney $U$ test was used to compare the TTS and PTS results between the groups. Repeated-measures analysis of variance (ANOVA) was used for time-dependent measurements to compare the PTA and TM test results and VAS, THI, and BDI scores before and after the MRI scans between the groups. Pearson or Spearman correlation analyses and chi-square or Fisher's exact tests were performed to determine the relationships between the VAS, THI, and BDI 
results and PTA and TM results. A two-sided $P$ value of $\leq 0.05$ was deemed significant.

\section{RESULTS}

The background noise in the room was $52.8 \mathrm{dBA}$ (58.3 dB) on average. The mean intensity of the acoustic noise during the MRI scan was recorded as $99.3 \pm 3.4 \mathrm{dBA}(109.9 \pm 4.1 \mathrm{~dB})$. Higher noise levels with a peak of $118 \mathrm{dBA}(126 \mathrm{~dB})$ were recorded as well, especially when the slice thickness declined and the coronal planes were examined.

A total of 63 symptomatic ears of 55 patients (mean age $56.3 \pm 10.3$ years, 38 males and 17 female) were included in the analyses before and after the MRI procedure. The patients were divided into two age groups, i.e., $<65$ years old ( $n=33$ ears of 29 patients) and $\geq 65$ years old ( $n=30$ ears of 26 patients). The left ear was symptomatic in $57.58 \%$ $(19 / 33)$ and $53.3 \%(16 / 30)$ of ears in patients aged $<65$ and $\geq 65$, respectively. Eight patients had bilateral tinnitus.

Moreover, TTS and PTS in the PTA hearing thresholds were observed in $28.6 \%(18 / 63)$ and $23.8 \%(15 / 63)$ of the ears, respectively. The highest threshold shifts within 24 hours after the MRI were observed as $15 \mathrm{~dB}$ at $4 \mathrm{kHz}$ and $25 \mathrm{~dB}$ at $8 \mathrm{kHz}$. In one patient, the frequency of tinnitus changed from $2,000 \mathrm{~Hz}$ to $4,000 \mathrm{~Hz}$.

One patient (a 70-year-old male) with left-sided tinnitus developed right-sided tinnitus just after an MRI with a loudness of $55 \mathrm{~dB}$ at a frequency of $8,000 \mathrm{~Hz}$. The patient developed PTS of $10 \mathrm{~dB}$, $10 \mathrm{~dB}$, and $15 \mathrm{~dB}$ for the 2,000, 4,000, and 8,000 $\mathrm{Hz}$ frequencies. Additionally, the tinnitus remained persistent with a loudness of $40 \mathrm{~dB}$ at an $8,000-\mathrm{Hz}$ frequency 1 month after the MRI.

Another patient (a 57-year-old male) with bilateral tinnitus of $45 \mathrm{~dB}$ at $6,000 \mathrm{~Hz}$ for the left side and $75 \mathrm{~dB}$ at $8,000 \mathrm{~Hz}$ for the right side developed a temporary rise of $30 \mathrm{~dB}$ and a permanent rise of $20 \mathrm{~dB}$ in tinnitus intensity for the right side after the MRI. The loudness of his tinnitus was $65 \mathrm{~dB}$ and $70 \mathrm{~dB}$ for the left and right ears, respectively, 1 month after the MRI.

Table 1 compares the audiometric measurements before and after 24 hours and 1 month after the MRI, as well as the self-reported questionnaire results before and 24 hours after the MRI between the patients in the two age groups. Almost all PTA thresholds were significantly different between the two age groups, whereas the tinnitus loudness and VAS, BDI, and THI scores were not (Table 1).

Table 2 depicts the comparisons of the mean PTA, TM thresholds, and VAS, BDI, and THI scores across time within each age group and between the two age groups ( $<65$ versus $\geq 65$ years) before and after the MRI scan. Significant deteriorations were observed in the PTA thresholds and tinnitus intensity and related disturbances in the pairedsample t-test, especially in the patients aged $\geq 65$ years. The tinnitus loudness displayed a significant rise from baseline in the symptomatic ear for both groups following the MRI scan $(p<0.001)$. While the time-dependent effects were significant for the PTA thresholds, the TM for loudness thresholds and self-reported tinnitus-related questionnaire scores were not statistically different before and after the MRI scan between the groups (Table 2), except for VAS 5 (item regarding sleep disturbance caused by tinnitus).

The baseline hearing thresholds were significantly different between the two age groups; therefore, analyses of threshold shifts were needed as well (Table 3). The TTSs (PTA threshold on the first day post-MRI minus baseline) and the PTSs (PTA threshold at the first month post-MRI minus baseline) differed statistically between patients aged $<65$ and aged $\geq 65$ for frequencies of 2000 and $4000 \mathrm{~Hz}(p=0.012, p=0.004$ for 2,000 $\mathrm{Hz}$ respectively; $\mathrm{p}=0.006, p=0.004$ for $4,000 \mathrm{~Hz}$ respectively). Whereas the mean PTA threshold shifts at $8,000 \mathrm{~Hz}$ were not statistically different 
Table 1. Comparison of basal and post-MRI audiometric and self-reported questionnaire results between the patients with tinnitus according to the age groups.

\begin{tabular}{|c|c|c|c|c|c|}
\hline & \multicolumn{2}{|c|}{ Age $<65$ years } & \multicolumn{2}{|c|}{ Age $\geq 65$ years } & \\
\hline \multicolumn{6}{|c|}{ Baseline Audiometry } \\
\hline & Mean \pm SD & Minimum-Maximum & Mean \pm SD & Minimum-Maximum & $p^{*}$ \\
\hline \multicolumn{6}{|l|}{ Air Conductance } \\
\hline $500 \mathrm{~Hz}$ & $14.24 \pm 12.76$ & $5-65$ & $20.33 \pm 7.18$ & $10-35$ & 0.025 \\
\hline $1000 \mathrm{~Hz}$ & $14.70 \pm 11.86$ & $5-70$ & $24.33 \pm 8.58$ & $15-45$ & 0.001 \\
\hline $2000 \mathrm{~Hz}$ & $18.64 \pm 13.42$ & $5-60$ & $28.33 \pm 10.99$ & $10-50$ & 0.002 \\
\hline $4000 \mathrm{~Hz}$ & $34.85 \pm 25.14$ & $5-95$ & $46.00 \pm 18.69$ & $15-85$ & 0.052 \\
\hline $8000 \mathrm{~Hz}$ & $42.42 \pm 25.04$ & $10-100$ & $57.33 \pm 16.85$ & $20-85$ & 0.008 \\
\hline Tinnitus Loudness & $49.85 \pm 16.56$ & $20-95$ & $50.83 \pm 19.08$ & $0-90$ & 0.827 \\
\hline \multicolumn{6}{|c|}{ Post-MRI (24 Hours) Audiometry } \\
\hline & Mean \pm SD & Minimum-Maximum & Mean \pm SD & Minimum-Maximum & $p^{*}$ \\
\hline \multicolumn{6}{|l|}{ Air Conductance } \\
\hline $500 \mathrm{~Hz}$ & $14.24 \pm 12.75$ & $5-65$ & $20.33 \pm 7.18$ & $10-35$ & 0.025 \\
\hline $1000 \mathrm{~Hz}$ & $14.70 \pm 11.86$ & $5-70$ & $24.33 \pm 8.58$ & $15-45$ & 0.001 \\
\hline $2000 \mathrm{~Hz}$ & $19.24 \pm 13.47$ & $5-60$ & $31.50 \pm 11.61$ & $10-55$ & $<0.001$ \\
\hline $4000 \mathrm{~Hz}$ & $35.15 \pm 26.18$ & $5-95$ & $50.17 \pm 21.31$ & $15-90$ & 0.016 \\
\hline $8000 \mathrm{~Hz}$ & $45.30 \pm 25.12$ & $10-100$ & $63.83 \pm 19.68$ & $20-90$ & 0.002 \\
\hline Tinnitus Loudness & $54.85 \pm 17.48$ & $30-100$ & $61.00 \pm 18.12$ & $35-105$ & 0.175 \\
\hline \multicolumn{6}{|c|}{ Post-MRI (1 month) Audiometry } \\
\hline & Mean \pm SD & Minimum-Maximum & Mean \pm SD & Minimum-Maximum & $p^{*}$ \\
\hline \multicolumn{6}{|l|}{ Air Conductance } \\
\hline $500 \mathrm{~Hz}$ & $14.24 \pm 12.75$ & $5-65$ & $20.33 \pm 7.18$ & $10-35$ & 0.025 \\
\hline $1000 \mathrm{~Hz}$ & $14.70 \pm 11.86$ & $5-70$ & $24.33 \pm 8.58$ & $15-45$ & 0.001 \\
\hline $2000 \mathrm{~Hz}$ & $18.64 \pm 13.42$ & $5-60$ & $29.83 \pm 10.30$ & $10-50$ & $<0.001$ \\
\hline $4000 \mathrm{~Hz}$ & $34.70 \pm 25.76$ & $5-95$ & $49.00 \pm 20.57$ & $15-90$ & 0.019 \\
\hline $8000 \mathrm{~Hz}$ & $44.70 \pm 25.52$ & $10-100$ & $63.17 \pm 19.63$ & $20-90$ & 0.002 \\
\hline Tinnitus Loudness & $52.27 \pm 16.34$ & $20-95$ & $56.50 \pm 17.92$ & $30-100$ & 0.335 \\
\hline \multicolumn{6}{|c|}{ Basal Self-report Tests } \\
\hline & Mean \pm SD & Minimum-Maximum & Mean \pm SD & Minimum-Maximum & $\mathrm{p}^{*}$ \\
\hline VAS 1 & $4.91 \pm 1.99$ & $2-9$ & $4.53 \pm 2.40$ & $0-10$ & 0.500 \\
\hline VAS 2 & $4.82 \pm 1.86$ & $2-9$ & $4.33 \pm 1.88$ & $0-10$ & 0.308 \\
\hline VAS 3 & $4.94 \pm 2.08$ & $2-9$ & $4.60 \pm 2.86$ & $0-10$ & 0.589 \\
\hline VAS 4 & $3.79 \pm 1.83$ & $1-7$ & $3.63 \pm 1.87$ & $0-7$ & 0.741 \\
\hline VAS 5 & $3.55 \pm 1.94$ & $1-7$ & $3.17 \pm 2.63$ & $0-9$ & 0.515 \\
\hline $\mathrm{BDI}$ & $3.24 \pm 4.58$ & $0-19$ & $4.30 \pm 2.78$ & $0-9$ & 0.278 \\
\hline $\mathrm{THI}$ & $33.33 \pm 19.54$ & $0-74$ & $35.67 \pm 14.60$ & $11-68$ & 0.596 \\
\hline \multicolumn{6}{|c|}{ Post-MRI (24 Hours) Self-report Tests } \\
\hline & Mean \pm SD & Minimum-Maximum & Mean \pm SD & Minimum-Maximum & $p^{*}$ \\
\hline VAS 1 & $5.33 \pm 2.38$ & $2-10$ & $5.07 \pm 2.07$ & $3-10$ & 0.638 \\
\hline VAS 2 & $5.61 \pm 2.34$ & $2-10$ & $5.10 \pm 1.37$ & $3-10$ & 0.306 \\
\hline VAS 3 & $5.82 \pm 2.63$ & $2-10$ & $4.93 \pm 2.23$ & $2-9$ & 0.157 \\
\hline VAS 4 & $4.48 \pm 1.99$ & $2-8$ & $4.13 \pm 2.27$ & $1-9$ & 0.515 \\
\hline VAS 5 & $4.79 \pm 2.40$ & $1-9$ & $3.73 \pm 2.52$ & $1-8$ & 0.094 \\
\hline $\mathrm{BDI}$ & $4.06 \pm 5.11$ & $0-21$ & $6.10 \pm 3.67$ & $0-12$ & 0.063 \\
\hline $\mathrm{THI}$ & $37.00 \pm 21.85$ & $4-84$ & $40.40 \pm 15.83$ & $14-84$ & 0.486 \\
\hline
\end{tabular}

BDI: Beck Depression Inventory, MRI: Magnetic Resonance Imaging, SD: Standard Deviation, THI: Tinnitus Handicap Inventory, VAS: Visual Analogue Scale. $p^{*}$ : Student's T-test. 
Table 2. Comparisons of mean thresholds in audiometric tests and mean scores in self-report questionnaires across time within each age group and between two age groups before and after MRI scan.

\begin{tabular}{|c|c|c|c|c|c|c|c|c|c|}
\hline & \multicolumn{3}{|c|}{ Group 1 (Age<65) } & \multirow[b]{2}{*}{$\mathrm{P}^{\star}$} & \multicolumn{3}{|c|}{ Group 2 (Age $\geq 65$ ) } & \multirow[b]{2}{*}{$p^{\star}$} & \multirow[b]{2}{*}{$p^{\star \star}$} \\
\hline & $\begin{array}{r}\text { Baseline } \\
(\mathrm{mean} \pm \mathrm{SD}) \\
\end{array}$ & $\begin{array}{r}1^{\text {st }} \text { day } \\
(\text { mean } \pm S D)\end{array}$ & $\begin{array}{r}1^{\text {st }} \text { month } \\
(\text { mean } \pm S D) \\
\end{array}$ & & $\begin{array}{r}\text { Baseline } \\
(\text { mean } \pm S D) \\
\end{array}$ & $\begin{array}{r}1^{\text {st }} \text { day } \\
(\text { mean } \pm S D)\end{array}$ & $\begin{array}{r}1^{\text {st }} \text { month } \\
(\text { mean } \pm S D) \\
\end{array}$ & & \\
\hline \multicolumn{10}{|l|}{ PTA (dB) } \\
\hline $500 \mathrm{~Hz}$ & $14.24 \pm 12.76$ & $14.24 \pm 12.75$ & $14.24 \pm 12.75$ & - & $20.33 \pm 7.18$ & $20.33 \pm 7.18$ & $20.33 \pm 7.18$ & - & 0.025 \\
\hline $1 \mathrm{kHz}$ & $14.70 \pm 11.86$ & $14.70 \pm 11.86$ & $14.70 \pm 11.86$ & - & $24.33 \pm 8.58$ & $24.33 \pm 8.58$ & $24.33 \pm 8.58$ & - & 0.001 \\
\hline $2 \mathrm{kHz}$ & $18.64 \pm 13.42$ & $19.24 \pm 13.47$ & $18.64 \pm 13.42$ & 0.044 & $28.33 \pm 10.99$ & $31.50 \pm 11.61$ & $29.83 \pm 10.30$ & 0.001 & 0.001 \\
\hline $4 \mathrm{kHz}$ & $34.85 \pm 25.14$ & $35.15 \pm 26.18$ & $34.70 \pm 25.76$ & 0.535 & $46.00 \pm 18.69$ & $50.17 \pm 21.31$ & $49.00 \pm 20.57$ & 0.001 & 0.024 \\
\hline $8 \mathrm{kHz}$ & $42.42 \pm 25.04$ & $45.30 \pm 25.12$ & $44.70 \pm 25.52$ & 0.001 & $57.33 \pm 16.85$ & $63.83 \pm 19.68$ & $63.17 \pm 19.63$ & 0.000 & 0.003 \\
\hline \multicolumn{10}{|l|}{$\mathrm{TM}(\mathrm{dB})$} \\
\hline Loudness & $49.85 \pm 16.56$ & $54.85 \pm 17.48$ & $52.27 \pm 16.34$ & $<0.001$ & $50.83 \pm 19.08$ & $61.00 \pm 18.12$ & $56.50 \pm 17.92$ & $<0.001$ & 0.385 \\
\hline
\end{tabular}

PTA: Pure Tone Audiometry, TM: Tinnitus Matching; SD: Standard Deviation.

Paired samples T-test, within each group, between the baseline and the first month measurements $\left(p^{\star}\right)$.

Repeated measures ANOVA (group $\times$ timexfrequency), between the subject effects $\left(p^{\star \star}\right)$.

\begin{tabular}{|r|r|r|r|r|r|r|r|}
\hline & $\begin{array}{r}\text { Baseline } \\
(m e a n \pm S D)\end{array}$ & $\begin{array}{r}\text { 1st day } \\
(m e a n \pm S D)\end{array}$ & $P^{*}$ & $\begin{array}{r}\text { Baseline } \\
(m e a n \pm S D)\end{array}$ & $\begin{array}{r}1 \text { st day } \\
\text { (mean } \pm \text { SD })\end{array}$ & $P^{* *}$ \\
\hline Test & & & & & & \\
\hline VAS 1 & $4.91 \pm 1.99$ & $5.33 \pm 2.38$ & 0.011 & $4.53 \pm 2.40$ & $5.07 \pm 2.07$ & 0.011 & 0.558 \\
\hline VAS 2 & $4.82 \pm 1.86$ & $5.61 \pm 2.34$ & $<0.001$ & $4.33 \pm 1.88$ & $5.10 \pm 1.37$ & $<0.001$ & 0.288 \\
\hline VAS 3 & $4.94 \pm 2.08$ & $5.82 \pm 2.63$ & 0.007 & $4.60 \pm 2.86$ & $4.93 \pm 2.23$ & 0.134 & 0.304 \\
\hline VAS 4 & $3.79 \pm 1.83$ & $4.48 \pm 1.99$ & 0.005 & $3.63 \pm 1.87$ & $4.13 \pm 2.27$ & 0.007 & 0.601 \\
\hline VAS 5 & $3.55 \pm 1.94$ & $4.79 \pm 2.40$ & $<0.001$ & $3.17 \pm 2.63$ & $3.73 \pm 2.52$ & $<0.001$ & 0.012 \\
\hline BDI & $3.24 \pm 4.58$ & $4.06 \pm 5.11$ & $<0.001$ & $4.30 \pm 2.78$ & $6.10 \pm 3.67$ & $<0.001$ & 0.130 \\
\hline THI & $33.33 \pm 19.54$ & $37.00 \pm 21.85$ & $<0.001$ & $35.67 \pm 14.60$ & $40.40 \pm 15.83$ & $<0.001$ & 0.535 \\
\hline
\end{tabular}

BDI: Beck Depression Inventory, SD: Standard Deviation, THI: Tinnitus Handicap Inventory, VAS: Visual Analogue Scale.

Paired samples T-test, within each group, between the baseline and the first day measurements $\left(p^{\star}\right)$.

Repeated measures ANOVA (group ×timexfrequency), between the subject effects $\left(p^{\star *}\right)$.

between the two age groups $(p=0.271, p=0.363$ for TTS and PTS, respectively). The mean TTSs between tinnitus loudness on the first day postMRI and baseline were $5.00 \pm 6.495 \mathrm{~dB}$ and 10.17 $\pm 11.179 \mathrm{~dB}$ for patients aged $<65$ and aged $\geq 65$ respectively; this difference was statistically significant $(p=0.018)$. However, this significance disappeared for PTSs $(2.42 \pm 5.019 \mathrm{~dB}$ and 5.67 $\pm 7.739 \mathrm{~dB}$ for patients aged $<65$ and $\geq 65$, respectively) ( $p=0.063)$.

When Table 4 was examined, it revealed that the frequency of TTS and PTS at PTA that occurred from baseline to post-MRI measurements was higher for the geriatric group (36.7\% vs. $21.2 \%$ for
TTS and $33.3 \%$ vs. $15.2 \%$ for PTS, in patients aged $\geq 65$ and aged $<65$ respectively), even though the difference was not statistically significant. The results were similar for the TM test for loudness, i.e., the frequency of TTS and PTS was higher for the geriatric group (50\% vs. $27.3 \%$ for TTS and $26.7 \%$ vs. $12.1 \%$ for PTS in patients aged $\geq 65$ and $<65$, respectively).

We defined a level change as follows: at least a one-point increase on a minimum of three items for the VAS; a $\geq 1$ level class change for the BDI (minimal, mild, moderate, and severe); and $a \geq 1$ level class change for the THI with respect to the functional, emotional, and catastrophic classes. 
Table 3. Comparison of mean threshold shifts in pure tone audiometry between two age groups of patients with tinnitus.

\begin{tabular}{|c|c|c|c|c|c|}
\hline & & Age $<65$ years & & Age $\geq 65$ years & \\
\hline \multicolumn{6}{|c|}{ Temporary Threshold Shift } \\
\hline & Mean \pm SD & Minimum-Maximum & Mean \pm SD & Minimum-Maximum & $p^{*}$ \\
\hline \multicolumn{6}{|l|}{ PTA Frequency } \\
\hline $500 \mathrm{~Hz}$ & $0 \pm 0.00$ & $0-0$ & $0 \pm 0.00$ & $0-0$ & 1.000 \\
\hline $1000 \mathrm{~Hz}$ & $0 \pm 0.00$ & $0-0$ & $0 \pm 0.00$ & $0-0$ & 1.000 \\
\hline $2000 \mathrm{~Hz}$ & $0.61 \pm 1.66$ & $0-5$ & $3.17 \pm 4.64$ & $0-15$ & 0.012 \\
\hline $4000 \mathrm{~Hz}$ & $0.76 \pm 2.21$ & $0-10$ & $4.17 \pm 5.89$ & $0-20$ & 0.006 \\
\hline $8000 \mathrm{~Hz}$ & $3.33 \pm 4.95$ & $0-15$ & $6.33 \pm 8.60$ & $0-25$ & 0.271 \\
\hline \multicolumn{6}{|c|}{ Permanent Threshold Shift } \\
\hline & Mean \pm SD & Minimum-Maximum & Mean \pm SD & Minimum-Maximum & $p^{*}$ \\
\hline \multicolumn{6}{|l|}{ PTA Frequency } \\
\hline $500 \mathrm{~Hz}$ & $0 \pm 0.00$ & $0-0$ & $0 \pm 0.00$ & $0-0$ & 1.000 \\
\hline $1000 \mathrm{~Hz}$ & $0 \pm 0.00$ & $0-0$ & $0 \pm 0.00$ & $0-0$ & 1.000 \\
\hline $2000 \mathrm{~Hz}$ & $0 \pm 0.00$ & $0-0$ & $1.50 \pm 2.98$ & $0-10$ & 0.004 \\
\hline $4000 \mathrm{~Hz}$ & $0.30 \pm 1.21$ & $0-5$ & $3.00 \pm 4.66$ & $0-15$ & 0.004 \\
\hline $8000 \mathrm{~Hz}$ & $2.73 \pm 4.16$ & $0-15$ & $5.67 \pm 8.28$ & $0-25$ & 0.363 \\
\hline
\end{tabular}

PTA: Pure Tone Audiometry, SD: Standard Deviation. p*: Mann-Whitney U test.

The rates of level changes were $43.3 \%$ for the VAS, $10 \%$ for the BDI, and $33.3 \%$ for the THI for geriatric patients. However, the incidence of self-report test deteriorations was not statistically significantly different between the two patient age groups ( $p$ $>0.05$ for all).

In the correlation analysis, the continuous variables of the PTA thresholds at the 4,000 and $8,000 \mathrm{~Hz}$ frequencies were strongly correlated with the VAS (especially VAS 1 and 5), BDI, and THI scores at the 0.01 level of significance (two-tailed). The mean THI score shift was correlated with the mean threshold shift in the PTA at the 2,000, 4,000, and $8,000 \mathrm{~Hz}$ frequencies before and after the MRI $(r=0.319, p=0.012 ; r=374, p=0.003 ; r=417$, $p=0.001$ for TTS at 2,000, 4,000, and $8,000 \mathrm{~Hz}$, respectively, and $r=0.294, p=0.019 ; r=350, p$ $=0.005 ; r=470, p<0.00$ for PTS at 2,000, 4,000, and $8,000 \mathrm{~Hz}$, respectively). Interestingly, the PTA threshold shifts at $8,000 \mathrm{~Hz}$ for TTS plus both 4,000 and $8,000 \mathrm{~Hz}$ for PTS were correlated with the THI score shifts in the geriatric group, whereas the correlations were significant only for TTS in the PTA at $4,000 \mathrm{~Hz}$ in patients aged $<65$. However, the positive correlations between the $\mathrm{THI}$ and $\mathrm{TM}$ threshold shifts from baseline were statistically significant in patients aged $<65(r=439, p=$ 0.011 for TTS, and $r=0.362, p=0.039$ for PTS), in contrast to geriatric patients ( $p>0.05$ ).

\section{DISCUSSION}

The most important outcome of the current study was that geriatric patients were more vulnerable to MRI-related noise induced hearing threshold changes. However, they did not tend to acknowledge this effect in the self-report questionnaires as much, which might be due to their decreased attention to the directed questions and diminished mental cooperation with these subjective tests.

Although the majority of the time-dependent 
Table 4. Descriptive analysis regarding the presence of temporary and permanent threshold shifts in audiometric tests and the presence of level changes in self-report questionnaires between the age groups.

\begin{tabular}{|c|c|c|c|c|c|}
\hline & & \multicolumn{2}{|c|}{ Age Group } & \multirow[b]{2}{*}{ n (total) } & \multirow[b]{2}{*}{$p$} \\
\hline & & Age $<65(\%)$ & Age $\geq 65(\%)$ & & \\
\hline \multirow[t]{3}{*}{ TTS-PTA } & absent & $26(78.8)$ & $19(63.3)$ & 45 & \\
\hline & present & $7(21.2)$ & $11(36.7)$ & 18 & $0.175^{\star}$ \\
\hline & $\mathrm{n}$ (total) & 33 & 30 & & \\
\hline \multirow[t]{3}{*}{ PTS-PTA } & absent & $28(84.8)$ & $20(66.7)$ & 48 & \\
\hline & present & 5 (15.2) & $10(33.3)$ & 15 & $0.081^{* *}$ \\
\hline & $\mathrm{n}$ (total) & 33 & 30 & & \\
\hline \multirow[t]{3}{*}{ TTS-TM } & absent & $24(72.7)$ & $15(50)$ & 39 & \\
\hline & present & $9(27.3)$ & $15(50)$ & 24 & $0.064^{*}$ \\
\hline & n (total) & 33 & 30 & & \\
\hline \multirow[t]{3}{*}{ PTS-TM } & absent & $29(87.9)$ & $22(73.3)$ & 51 & \\
\hline & present & $4(12.1)$ & $8(26.7)$ & 12 & $0.126^{\star *}$ \\
\hline & $\mathrm{n}$ (total) & 33 & 30 & & \\
\hline \multirow[t]{3}{*}{ VAS-difference } & absent & $22(66.7)$ & $17(56.7)$ & 39 & \\
\hline & present & $11(33.3)$ & $13(43.3)$ & 24 & $0.414^{*}$ \\
\hline & $\mathrm{n}$ (total) & 33 & 30 & & \\
\hline \multirow[t]{3}{*}{ BDI-difference } & absent & $33(100)$ & $27(90)$ & 60 & \\
\hline & present & $0(0)$ & $3(10)$ & 3 & $0.102^{\star \star}$ \\
\hline & $\mathrm{n}$ (total) & 33 & 30 & & \\
\hline \multirow[t]{3}{*}{ THI-difference } & absent & $26(78.8)$ & $20(66.7)$ & 46 & \\
\hline & present & $7(21.2)$ & $10(33.3)$ & 17 & $0.279 *$ \\
\hline & n (total) & 33 & 30 & & \\
\hline
\end{tabular}

BDI: Beck Depression Inventory, PTA: Pure Tone Audiometry, PTS: Permanent Threshold Shift, THI: Tinnitus Handicap Inventory, TM: Tinnitus Matching, TTS: Temporary Threshold Shift, VAS: Visual Analogue Scale.

$p^{\star}$ : Chi-Square test, $p^{\star \star}$ : Fisher's Exact test.

analyses for the PTA, TM, VAS, BDI, and THI were insignificant and crosstabs for the presence of threshold shifts and level changes were indifferent statistically between two age groups, impairment was observed in the numbers and percentages in the patients aged $>65$ years after MRI.

Temporal bone MRI is required in patients with unilateral unexplained tinnitus with or without hearing loss, patients with bilateral symmetric and asymmetric hearing loss suspected of retrocochlear pathology, and patients with suspected intracranial tumors. During an MRI scan, various types of noise at different pitches and intensities occur. This leads to symptoms of discomfort for both patients and health care workers, including being unable to communicate, anxiety, claustrophobia, and hearing loss or any kind of acoustic trauma (3-5,12-14).

The sensitivity of the human ear to sound begins at $0 \mathrm{~dB}$ (hearing threshold), and disturbance occurs when the SPL exceeds 120-140 dB (pain threshold). In 1.5 Tesla MRI scans, 81-117 dB SPLs 
are common and may reach as high as $131 \mathrm{~dB}$ for high-speed echo planar imaging $(4,15)$. Both the auditory and vestibular labyrinths may be damaged by high acoustic sound levels. Golz et al. reported that unilateral or bilateral hearing loss might be associated with the abnormal vestibular functions that result from noise (16).

Several otological conditions, especially asymmetric hearing loss, fall under the etiology of tinnitus. Although otological anomalies may be the source of initial tinnitus, it is more likely to continue due to subsequent neural changes in the central auditory system $(17,18)$. While $17 \%$ of the general population has been reported to have tinnitus, this rate could be as high as $33 \%$ in the geriatric population (19). Presbycusis is responsible for the majority of the etiology of tinnitus, but acoustic trauma also holds a significant place.

In recent years, there has been a growing interest in MRI systems, which has resulted in an increased frequency of requested MRI examinations, which consequently might influence the acoustic characteristics of the inner ear and the incidence of MRI-induced hearing loss or tinnitus (20). The characteristics of the frequency spectra (generally between 0.2 and $1.5 \mathrm{kHz}$ ) of acoustic noise generated by MRI depend on the equipment composition and system protocol (21). In particular, Cho et al. (22) found that under loud noise (100 dB), the frequency range occurs in a wide range up to $4 \mathrm{kHz}$, with a peak at around 2.4 $\mathrm{Hz}$.

The present study also demonstrated that the acoustic noise induced by 1.5 Tesla MRI of the temporal region has a negative impact on the audiometric and self-reported quality measures of tinnitus. Previous studies have reported on depression or anxiety caused by MRI and the bidirectional relationship between such depression and MRI-induced tinnitus (12-14). Furthermore, herein, the MRI noise itself was detected at peak levels of $118 \mathrm{dBA}(126 \mathrm{~dB})$ to clearly address the cause-and-effect relationship with post-MRI tinnitus.

In patients with tinnitus, the severity is associated with the severity of anxiety and depression (23). The literature has shown that tinnitus causes more dissatisfaction in older patients, as it may alter sleep patterns and emotional states (24). In this study, sleep disturbances caused by tinnitus (VAS 5) were more evident in patients aged $<65$, which might be explained by the fact that sleep deprivation could be less tolerable for younger or more socially active people. The numeric values of all VAS scores were higher in patients aged $<65$ in contrast to the BDI and THI scores, which were higher in geriatric patients. Moreover, the frequency of deterioration on the BDI and $\mathrm{THI}$ scales was also higher for the latter group of patients in the present study.

To the best of our knowledge, this is the first study to address the threshold shifts in frequencyspecific hearing and tinnitus loudness together with self-reported tinnitus disturbance tests between geriatric and non-geriatric patients. Since exposure to acoustic noise has been found to cause hearing loss, even in a small group of patients, acoustic noise should be assumed to be one of the MRI safety-first parameters. Therefore, hearing protection should be required for all patients, geriatric or not, with the use of earplugs to avoid the undesired effects of this equipment.

\section{CONCLUSION}

It may be inferred from this study that 1.5 Tesla contrast-enhanced MRI of the temporal bone might cause deterioration of the hearing and increase tinnitus loudness thresholds and discomfort caused by tinnitus especially in geriatric patients. Therefore, we must consider further the indications of its necessity and take measures to protect the hearing of all patients undergoing an MRI scan. 


\section{REFERENCES}

1. Annesley-Williams DJ, Laitt D, Jenkins JP, Ramsden RT, Gillespie JE. MRI in the investigation of sensorineural hearing loss: is contrast enhancement still necessary? J Laryngol Otol 2001;115(1):14-21. (PMID:11233615)

2. Revadi G, Rahmat $\mathrm{O}$, Raman $\mathrm{R}$, Norlisah $\mathrm{R}, \mathrm{Ng} \mathrm{KH}$. Hearing loss after noise exposure. Auris Nasus Larynx 2011;38(4):519-22. (PMID:21236610)

3. Brummett RE, Talbot JM, Charuhas P. Potential hearing loss resulting from MR imaging. Radiology 1988;169(2):539-40. (PMID:3175004)

4. McJury M, Blug A, Joerger C, Condon B, Wyper D. Short communication: acoustic noise levels during magnetic resonance imaging scanning at $1.5 \mathrm{~T}$. Br J Radiol 1994;67(796):413-5. (PMID:8173889)

5. Jin C, Li H, Li X, Wang M, Liu C, Guo J, Yang J. Temporary hearing threshold shift in healthy volunteers with hearing protection caused by acoustic noise exposure during 3-T multisequence. MR Neuroimaging. Radiology 2018;286(2):602-8. (PMID:28813235)

6. Govindaraju R, Omar R, Rajagopalan R, Norlisah $\mathrm{R}$, Kwan-Hoong $\mathrm{N}$. Hearing loss after noise exposure. Auris Nasus Larynx 2011;38(4):519-22. (PMID:21236610)

7. Lim EY, Tang IP, Peyman M, Ramli N, Narayanan P, Rajagopalan R. 3 Tesla magnetic resonance imaging noise in standard head and neck sequence does not cause temporary threshold shift in high frequency. Eur Arch Otorhinolaryngol 2015;272(11):3109-13. (PMID:25205300)

8. Figueiredo RR, Azevedo AA, Oliveira Pde M. Correlation analysis of the visual-analogue scale and the Tinnitus Handicap Inventory in tinnitus patients. Revista Brasileira de Otorrinolaringologia. 2009;75(1):76-9. (PMID:19488564)

9. Aksoy S, Firat Y, Alpar R. The Tinnitus Handicap Inventory: a study of validity and reliability. The international tinnitus journal 2007;13(2):94-8. (PMID:18229787)

10. Newman CW, Jacobson GP, Spitzer JB. Development of the Tinnitus Handicap Inventory. Arch Otolaryngol Head Neck Surg 1996;122(2):143-8. (PMID:8630207)

11. Beck AT, Steer RA, Brown GK. BDI-II: Beck depression inventory manual. 2nd edition, Psychological Corporation, San Antonio, TX, USA 1996.

12. Quirk ME, Letendre AJ, Ciottone RA, Lingley
JF. Anxiety in patients undergoing MR imaging. Radiology 1989;170(2):463-6. (PMID:2911670)

13. Kanal E, Shellock FG, Talagala L. Safety considerations in MR imaging. Radiology 1990;176(3):593-606. (PMID:2202008)

14. Shellock FG. Biological effects and safety aspects of magnetic resonance imaging. Magn Reson $\mathrm{Q}$ 1989;5(4):243-61. (PMID:2701284)

15. Amaro E, Jr, Williams SC, Shergill SS, et al. Acoustic noise and functional magnetic resonance imaging: current strategies and future prospects. J Magn Reson Imaging 2002;16(5):497-510. (PMID:12412026)

16. Golz A, Westerman ST, Westerman LM, et al. The effects of noise on the vestibular system. Am J Otolaryngol 2001;22(3):190-6. (PMID:11351289)

17. Eggermont JJ, Roberts LE. The neuroscience of tinnitus. Trends Neurosci 2004;27:676-82. (PMID:15474168)

18. Noreña AJ, Eggermont JJ. Changes in spontaneous neural activity immediately after an acoustic trauma: Implications for neural correlates of tinnitus. Hear Res 2003;183(1-2):137-53. (PMID:13679145)

19. NondahI DM, Cruickshanks KJ, Wiley TL, Klein R, Klein BE, Tweed TS, et al. Prevalence and 5-year incidence of tinnitus among older adults: The epidemiology of hearing loss study. J Am Acad Audiol 2002;13(6):32331. (PMID:12141389)

20. Strainer JC, Ulmer JL, Yetkin FZ, et al. Functional MR of the primary auditory cortex: an analysis of pure tone activation and tone discrimination. AJR 1997;18(4):601-10. (PMID:9127019)

21. Counter SA, Olofsson A, Grahn, Borg E. MRI acoustic noise: sound pressure and frequency analysis. J Magn Reson Imaging 1997;7(3):606-11. (PMID:9170052)

22. Cho ZH, Park SH, Kim JH, et al. Analysis of acoustic noise in MRI. Magn Reson Imaging 1997;15(7):815-22. (PMID:9309612)

23. Zöger S, Svedlund J, Holgers KM. Relationship between tinnitus severity and psychiatric disorders. Psychosomatics 2006;47(4):282-8. (PMID:16844885)

24. Ferreira LM, Ramos Junior AN, Mendes EP. Characterization of tinnitus in the elderly and its possible related disorders. Rev. Bras. Otorrinolaringol 2009;75(2):245-8. (PMID:19575111) 